\title{
Serum Alkaline Phosphatase in Hypophosphatasia
}

\author{
Joseph B. Warshaw, John W. Littlefield, William H. Fishman, \\ Norma R. INGLis, and Leo L. Stolbach \\ From the Children's Service, Massachusetts General Hospital; Shriners Burns \\ Institute and the Department of Pediatrics, Harvard Medical School; and the \\ Departments of Medicine and Pathology, Tufts University School of Medicine, \\ Boston, Massachusetts 02114
}

A B S T R A C T It is recognized that serum alkaline phosphatase may reflect enzyme contributions from bone, liver, and intestine. We have investigated serum alkaline phosphatases in two siblings with hypophosphatasia. After administration of long-chain triglycerides, the major alkaline phosphatase component of their sera was shown to be of intestinal origin on the basis of inhibition by $l$-phenylalanine. Starch block electrophoresis suggested that there were other regions of l-phenylalanine-sensitive alkaline phosphatase in addition to the major slow-moving intestinal band. Medium-chain triglycerides which are absorbed by the portal route did not cause a similar augmentation of intestinal alkaline phosphatase activity. These studies indicate that serum levels of intestinal alkaline phosphatase are increased normally after long-chain fat feeding in hypophosphatasia and may be the major component of total serum alkaline phosphatase activity.

\section{INTRODUCTION}

Hypophosphatasia is a disorder of skeletal growth first described by Rathbun (1) in 1943. Patients with this disease may have a spectrum of disability ranging from mild growth retardation to a crippling disorder associated with severe skeletal deformities (2). A clinical hallmark of this condition is premature loss of the deciduous teeth (3). Chemically, hypophosphatasia is characterized by low levels of serum alkaline phosphatase and increased amounts of phosphoethanolamine in the urine (4).

In recent years it has become apparent that total alkaline phosphatase activity in sera reflects a number of alkaline phosphohydrolases of several tissue origins. For example, tissue-specific alkaline phosphatases have

Dr. Fishman was supported by U. S. Public Health Service Career Research Award K6-CA-18453.

Received for publication 4 March 1971 and in revised form 7 April 1971. been identified in bone, liver, intestine, and placenta $(5,6)$. The precise metabolic role of these enzymes including that of bone remains obscure.

The enzymes have been differentiated in various tissues and in serum on the basis of their inhibition by different physical and chemical treatments; for example, the bone enzyme is the most heat labile. Intestinal alkaline phosphatase is neuraminidase resistant and is inhibited by $l$-phenylalanine $(5,7)$.

The present investigation was undertaken to delineate the contributions of the intestine and other tissues to the total serum alkaline phosphatase activity of patients with hypophosphatasia. It has been established that individuals with $\mathrm{O}$ or $\mathrm{B}$ blood groups, who secrete $\mathrm{ABH}$ substances, show a rise in serum intestinal alkaline phosphatase after eating (8). Danovitch, Baer and Laster (9) have demonstrated previously that intestinal biopsies obtained from patients with hypophosphatasia had normal alkaline phosphatase activity. The present study indicates that alkaline phosphatase of intestinal origin may be the major source of alkaline phosphatase activity in the serum of patients with hypophosphatasia.

\section{METHODS}

Routine serum alkaline phosphatase was determined in the hospital chemistry laboratory by the method of Bessey, Lowry, and Brock (10) and reported in Bodansky Units. Subsequently, alkaline phosphatase was also determined by an automated method as described by Fishman and Green (11) and reported in King-Armstrong Units. The latter method measures alkaline phosphatase activity in the presence of $d$-phenylalanine and $l$-phenylalanine. It has been shown that $5 \mathrm{~mm}$ l-phenylalanine in the assay inhibits intestinal alkaline phosphatase $77 \%$ whereas the $d$-isomer is without effect (11). A measure of the intestinal contribution to the total serum alkaline phosphatase is obtained by substracting the values obtained with $l$-phenylalanine in the assay from the total alkaline phosphatase (in the presence of $d$-phenylalanine) activity (12). Heat sensitivity of serum alkaline phosphatase is determined by heating the samples for $16 \mathrm{~min}$ at $55^{\circ} \mathrm{C}$ in a circulating water bath (5). 
Electrophoresis of serum was carried out on horizontal starch gel plates as described by Fishman and Ghosh (5). After the run, alkaline phosphatase was developed in a medium containing $0.3 \%$ sodium alpha naphthol phosphate in $50 \mathrm{~mm}$ carbonate-bicarbonate buffer, $\mathrm{pH} 9.8$ for $1 \mathrm{hr}$ at $37^{\circ} \mathrm{C}$. The gels were then washed and immersed for $1 \mathrm{hr}$ in a bath containing the diazo dye, Fast Blue RR $(0.1 \%$ in $50 \mathrm{~mm}$ carbonate-bicarbonate buffer, $\mathrm{pH} 10$ ).

Routine blood chemistries were performed by the hospital chemistry laboratory. Phosphoethanolamine concentration in urine was determined by Dr. Vivian Shih using the method of Efron (13). Leukocyte alkaline phosphatase was determined by the method of Beck and Valentine (14).

\section{CLINICAL SUMMARIES}

Case I. D. R. was first admitted to the Children's Service of the Massachusetts General Hospital at 2 months of age for treatment of leg deformities present since birth. He was the second child delivered by cesarean section to a healthy mother. The birth weight was $6 \mathrm{lb} .12 \mathrm{oz}$. He was an active infant with striking malformations of his extremities. The patient had varus deformities of both feet, and the femora and tibiae were both angulated bilaterally. Bony spurs were noted on the anterior aspects of his tibiae. Small cutaneous dimples were superficial to these spurs bilaterally. These dimples were thought to be the result of intrauterine fractures. There was also bowing of both forearms. A small umbilical hernia was noted. The neurological examination was normal. The hematocrit was $20 \%$ and the white blood count 9700 with a normal differential. The patient secreted $\mathrm{H}$ substance and was of blood group $\mathrm{B}$. The urinalysis was normal. The serum concentration of calcium was $9.7 \mathrm{mg} / 100 \mathrm{ml}$, phosphorous $7.8 \mathrm{mg} / 100$ $\mathrm{ml}$, blood urea nitrogen $9 \mathrm{mg} / 100 \mathrm{ml}$, creatinine $0.4 \mathrm{mg} /$ $100 \mathrm{ml}$. The alkaline phosphatase was 2.0 Bodansky U. The protein-bound iodine and the serum protein electrophoresis were within normal limits. The amino acid pattern in both blood and urine was also normal at that time. X-ray evaluation showed the bones of the calvaria to be unusually thin. No skull fractures were noted.

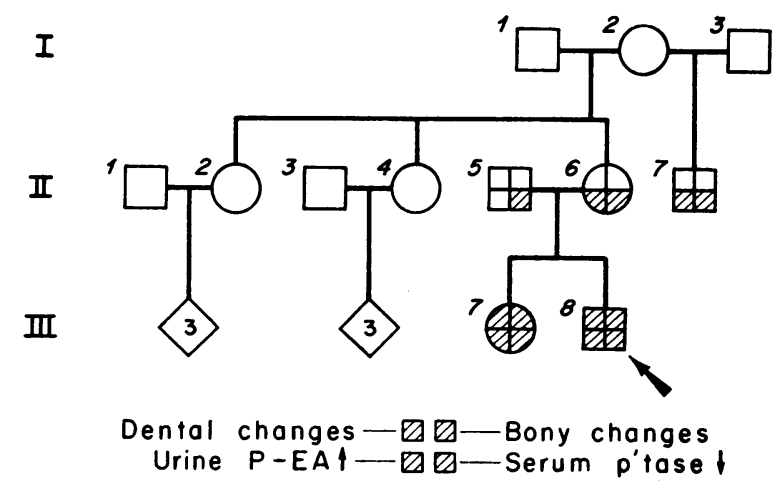

Figure 1 Pedigree of family.
The bones of the pelvis and trunk appeared to be more lucent than normal, and radiographs of his extremities showed them to be grossly deformed with features consistent with intrauterine fractures.

The patient underwent open corrective osteotomies of his right femur and left tibia and fibula. At that time the presumptive diagnosis was osteogenesis imperfecta although the low value for serum alkaline phosphatase also suggested the diagnosis of hypophosphatasia.

At 3 months of age he was readmitted for correction of a left inguninal hernia and hydrocele. He had a number of subsequent admissions for corrective orthopedic procedures, and at 26 months of age he underwent correction of craniosynostosis involving the coronal and lamboid sutures.

His performance level has remained consistent with his chronological age, and he has had no evidence of neurological deficit. He has remained below the 3rd percentile for both height and weight. The deciduous teeth erupted at 9 months of age but began to shed at 14 months of age. At 36 months of age, only one premolar remained.

The serum alkaline phosphatase has remained low, and except for the normal initial values, the urine phosphoethanolamine has remained in the range consistent with the diagnosis of hypophosphatasia. Leukocyte alkaline phosphase ranged from 14.9 to $29.8 \mathrm{U}$ (normal is $15-35$ $\mathrm{U}$ with $1 \mathrm{U}$ equaling $1 \mathrm{mg}$ of phosphorus liberated per $10^{10}$ myeloid cells per $\mathrm{hr}$ ). The increased level of urinary phosphoethanolamine may have been missed initially because phosphoethanolamine may be masked by taurine on a screening amino acid chromatogram.

Case II. M. R. is the older female sibling of D. R. She weighed $7 \mathrm{lb} .8 \mathrm{oz}$ at birth and was delivered by cesarean section because of impending eclampsia. Her deciduous teeth erupted at an appropriate age but had begun to shed by the time she was 15 months of age. Developmental milestones were normal, but her weight and height have been below the 3rd percentile for her age. The serum alkaline phosphatase was 1.7 Bodansky $U$. The serum calcium was $9.2 \mathrm{mg} / 100 \mathrm{ml}$, and the phosphorus was $6.2 \mathrm{mg} / 100 \mathrm{ml}$. The leukocyte alkaline phosphatase varied from 9.7 to $29.8 \mathrm{U}$. On physical examination she was noted to have short stature, a waddling gait, and had lost all but three deciduous teeth. There were no gross skeletal malformations, but she did have beading of the costochondral junctions. She was also a secreter of $\mathrm{H}$ substance and of $\mathrm{B}$ blood type.

Family history. The pedigree for the immediate members of the family is given in Fig. 1. Except for III 7 and III 8 , there is no history of early loss of teeth, shortness of stature, or other bony abnormalities. No further physical or radiographic examinations have been done, and no chemical analyses have been obtained except 
for those in Table I. Presumably, I 2 is a heterozygote, and II 6 and II 7 (both affected only chemically) are presumably heterozygous, too. II 5 is unrelated to II 6 and probably also heterozygous despite the absence of phosphoethanolamine in his urine $(2,15)$. II 5 may carry a different allele for "less severe" hypophosphatasia or expression of the heterozygous state may be variable. The disorder is known to be biochemically heterogeneous. In any case the disorder is probably behaving as an autosomal recessive in this family; this is the mode of inheritance usually described although probable dominant inheritance has twice been reported $(9,16)$.

Both patients were subsequently readmitted for the studies outlined below. At the time of admission D. R. and M. R. were 4 and $6 \mathrm{yr}$ of age, respectively.

l-Phenylalanine-sensitive serum alkaline phosphatase in hypophosphatasia. Upon admission, the children were placed on a low fat diet (less than $5 \%$ fat) and on the following morning (day 2), they were given $240 \mathrm{ml}$ of $3200 \mathrm{AH}$ formula (Mead Johnson \& Co., Evansville, Ind.) containing $7.5 \mathrm{~g}$ of medium-chain triglyceride (MCT). ${ }^{1}$ Blood samples were obtained before administration of $\mathrm{MCT}$ and again $7 \mathrm{hr}$ later. On the second morning 30 $\mathrm{g}$ of long-chain triglyceride (LCT) in the form of corn oil was administered, and serum samples for alkaline phosphatase determination and starch gel electrophoresis were again obtained before and $7 \mathrm{hr}$ after the administration of the LCT. The influence of the different lipid feedings on total serum alkaline phosphatase activity is shown in Table II. Admission serum alkaline phosphatase activity of both children decreased significantly after institution of the low fat diet. The administration of

${ }^{1}$ Abbreviations used in this paper: LCT, long-chain triglyceride; MCT, medium-chain triglyceride.
TABLE I

Serum Alkaline Phosphatase and Urine Phosphoethanolamine Levels

\begin{tabular}{|c|c|c|c|}
\hline Name & Date & $\begin{array}{c}\text { Alkaline } \\
\text { phosphatase }\end{array}$ & Phosphoethanolamine \\
\hline & & Bodansky units & $m g / 24 h r$ \\
\hline \multirow[t]{4}{*}{ II 5} & $5 / 68$ & 1.9 & 6.7 \\
\hline & $5 / 69$ & 1.4 & 8.6 \\
\hline & $6 / 69$ & 1.6 & \\
\hline & $10 / 69$ & 1.7 & \\
\hline \multirow[t]{2}{*}{ II 6} & $5 / 68$ & 1.2 & 33.6 \\
\hline & $3 / 69$ & 1.2 & 34.0 \\
\hline \multirow[t]{2}{*}{ II 7} & $9 / 68$ & 1.2 & \\
\hline & $4 / 69$ & 1.2 & 45.0 \\
\hline \multirow[t]{3}{*}{ III 7} & $8 / 68$ & 1.7 & 30.0 \\
\hline & $3 / 69$ & 1.7 & 53.2 \\
\hline & $4 / 69$ & 1.2 & \\
\hline \multirow[t]{6}{*}{ III 8} & $6 / 66$ & $2.8,2.1$ & \\
\hline & $12 / 67$ & $1.6,1.4$ & \\
\hline & $4 / 68$ & 1.2 & \\
\hline & $9 / 68$ & 1.0 & 100.8 \\
\hline & $3 / 69$ & 1.2 & 82.6 \\
\hline & $4 / 69$ & 1.0 & \\
\hline
\end{tabular}

Normal range for alkaline phosphatase in infants is $\mathbf{5 - 1 0}$ Bodansky $U$ and $2-4.5$ in adults. Normal urinary phosphoethanolamine excretion is approximately $3-13 \mathrm{mg} / 24 \mathrm{hr}(9)$.

MCT resulted in only a slight increase in total alkaline phosphatase activity (day 2, 3 p.m. sample), and activity again fell to a low level on continuation of the low fat diet (day 3, 8 a.m.). The increase of the total alkaline phosphatase activity after LCT administration, however, was quite striking. Both children showed ap-

TABLE II

The Effect of Lipid Feeding on Serum Alkaline Phosphatase Activity

\begin{tabular}{|c|c|c|c|c|c|c|}
\hline \multirow{2}{*}{$\frac{\text { Patient }}{\text { M. R. }}$} & \multicolumn{2}{|c|}{ Time } & \multirow{2}{*}{$\frac{\text { Diet }}{\text { Admission }}$} & \multirow{2}{*}{$\begin{array}{c}\begin{array}{c}\text { Total } \\
\text { activity }\end{array} \\
2.19\end{array}$} & \multirow{2}{*}{ 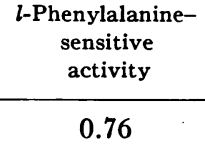 } & \multirow{2}{*}{$\frac{\begin{array}{c}\% \text { l-Phenylalanine } \\
\text { inhibition }\end{array}}{34.8}$} \\
\hline & Day 1 & 1 p.m. & & & & \\
\hline & Day 2 & 8 a.m. & Low fat & 1.54 & 0.41 & 26.3 \\
\hline & & 3 p.m. & MCT & 2.05 & 0.62 & 30.3 \\
\hline & Day 3 & 8 a.m. & Low fat & 1.40 & 0.17 & 11.9 \\
\hline & & 3 p.m. & LCT & 4.43 & 2.56 & 57.7 \\
\hline \multirow[t]{5}{*}{ D. R. } & Day 1 & 1 p.m. & Admission & 3.82 & 2.09 & 54.8 \\
\hline & Day 2 & 8 a.m. & Low fat & 1.49 & 0.16 & 10.7 \\
\hline & & 3 p.m. & MCT & 1.82 & 0.39 & 21.3 \\
\hline & Day 3 & 8 a.m. & Low fat & 1.35 & 0.17 & 12.5 \\
\hline & & 3 p.m. & LCT & 4.76 & 3.03 & 63.7 \\
\hline
\end{tabular}

Lipid feedings were given immediately after the 8 a.m. serum samples were obtained. Assays were carried out in the presence of $d$ - or $l$-phenylalanine as described in the text. 


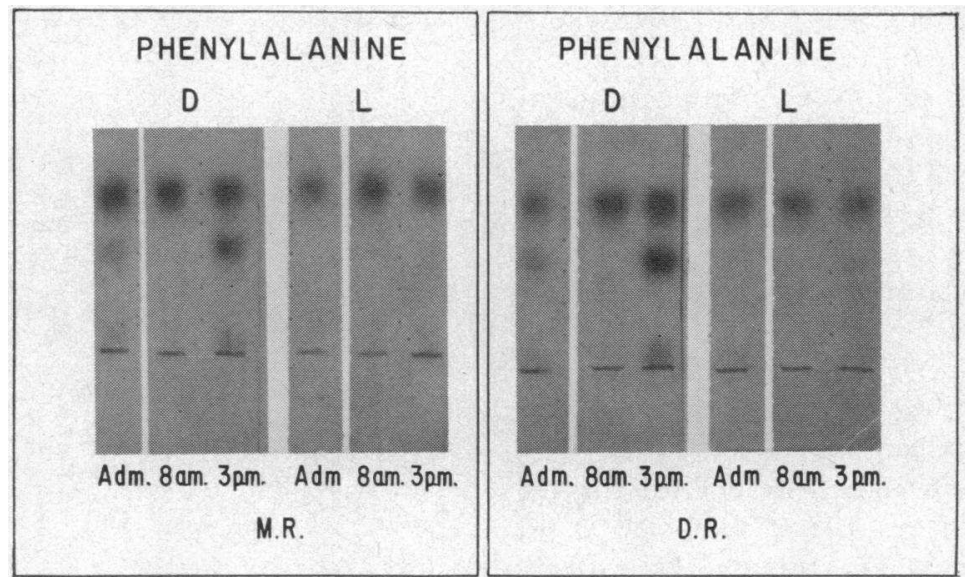

FIGURE 2 Serum alkaline phosphatase profiles after feedings of longchain triglycerides. Sera from patients $M$. R. and D. R. were incubated in the presence of $d$ - and $l$-phenylalanine before and after administration of LCT. The first samples were obtained upon admission (Adm). The 8 a.m. sample was obtained after an overnight fast and the 3 p.m. sample $7 \mathrm{hr}$ after administration of the LCT.

proximately a three fold increase in total alkaline phosphatase activity $7 \mathrm{hr}$ after LCT administration.

$l$-Phenylalanine inhibited a major fraction of the total serum alkaline phosphatase activity of these patients. As noted above, $l$-phenylalanine sensitivity is a measure of intestinal alkaline phosphatase activity. The $l$-phenylalanine-sensitive alkaline phosphatase fraction of these patients' sera decreased after institution of the low fat diet and was not stimulated significantly until LCT were administered. As is also seen in Table II, more than $50 \%$ of total alkaline phosphatase activity was $l$-phenylalanine-sensitive after LCT loading. It should be emphasized that the maximum inhibition of purified alkaline phosphatase by $l$-phenylalanine is $77 \%$ (11).

Electrophoretic profiles of the serum alkaline phosphatase components of both patients' sera are shown in Fig. 2. The starch block profiles of serum incubated in the presence of $d$-phenylalanine is seen on the left, and incubations carried out in the presence of the inhibitor $l$-phenylalanine are shown on the right. The admission sample incubated in the presence of 1-phenylalanine showed two major bands. After patients were placed on the low fat diet, the slower band, presumably of intestinal origin, disappeared. However, this band again became the dominant serum alkaline phosphatase component after the administration of LCT. Alkaline phosphatase activity was also detected at the origin of the gel. When incubations were carried out in the presence of $l$-phenylalanine, both the slower moving band and that at the origin disappeared. The fastest component thought to reflect alkaline phosphatase of skeletal and liver origin also decreased in intensity in the presence of $l$-phenylalanine suggesting that an intestinal alkaline phosphatase component was also present in that band

TABLE III

Heat Stability and l-Phenylalanine Sensitivity after Long-Chain Triglyceride (LCT) Administration

\begin{tabular}{lcccccc}
\hline & $\begin{array}{c}\text { Total } \\
\text { AP }\end{array}$ & $\begin{array}{c}\text { Heat- } \\
\text { sensitive } \\
\text { AP }\end{array}$ & $\begin{array}{c}\text { Heat-stable } \\
\text { AP }\end{array}$ & $\begin{array}{c}\text { \% AP inhibited } \\
\text { by heating }\end{array}$ & $\begin{array}{c}l \text {-Phenylalanine- } \\
\text { sensitive AP } \\
\text { after heating }\end{array}$ & $\begin{array}{c}\text { \% Inhibition by } \\
\text { l-phenylalanine } \\
\text { after heating }\end{array}$ \\
\hline $\begin{array}{l}\text { M. R. } \\
\text { Fasting }\end{array}$ & 1.40 & 0.93 & 0.47 & 66.4 & & \\
3 p.m. & 4.43 & 1.82 & 2.61 & 41.1 & 1.97 & 75.4 \\
D. R. & & & & & & \\
Fasting & 1.35 & 0.84 & 0.51 & 62.2 & 0.16 & 32.7 \\
3 p.m. & 4.76 & 1.20 & 3.56 & 25.3 & 2.47 & 73.5 \\
\hline
\end{tabular}

The LCT was given immediately after the fasting samples were obtained. Experimental conditions are as described in the text. AP = alkaline phosphatase. 
as well as at the origin of the gel. After administration of MCT, there was no increase in the band presumed to be of intestinal origin.

Heat stability of serum alkaline phosphatase. Alkaline phosphatase of intestinal origin shows considerable heat stability. In contrast, alkaline phosphatase of skeletal origin shows greater thermal lability than that of other tissue origins (17). The effects of heat treatment of the fasting alkaline phosphatase activity and that $7 \mathrm{hr}$ after administration of LCT are shown in Table III. Heat treatment of the patients' sera resulted in a loss of total alkaline phosphatase activity. In the fasted state, the heat-labile fraction accounted for about $60 \%$ of total serum alkaline phosphatase activity. There was a relative persistence of the $l$-phenylalanine-sensitive activity (intestinal alkaline phosphatase) after heat treatment. This was particularly evident after LCT administration. After LCT administration, the $l$-phenylalanine-sensitive fraction accounted for approximately $75 \%$ of the total alkaline phosphatase activity. Thus, virtually all of the alkaline phosphatase activity remaining after heating and the dominant serum fraction after LCT administration were of intestinal origin.

The effect of heating on the electrophoretic profiles of the patients in the fasting state and after administration of LCT is shown in Fig. 3. In the fasting state, only one fast-moving band was noted. This band was markedly decreased in intensity after heat exposure. The heat lability of the fast band suggests that the major fraction of that band is of skeletal origin (5). The residual activity in the fast band probably reflects alkaline phosphatase of hepatic origin and perhaps some intestinal fraction. The slower (intestinal) band seen after LCT administration was relatively stable after incubation of the serum at $55^{\circ} \mathrm{C}$ for $16 \mathrm{~min}$.

\section{DISCUSSION}

The presence of intestinal alkaline phosphatase activity in serum seems to relate to the $A B O$ blood groups and secretion of $\mathrm{ABH}$ substances (18). Individuals, who have the slower moving intestinal activity in their sera, are secreters of $\mathrm{ABH}$ blood group substances and are generally blood group $\mathrm{O}$ or B. Our patients were of blood group $\mathrm{B}$ and were secretors of $\mathrm{H}$ substance. Fatenriched feedings are known to increase alkaline phosphatase activity of intestinal lymph (19) and also to increase the intestinal component in serum of individuals who genetically show that activity $(8,20)$.

The present investigation documents that patients with hypophosphatasia can show a normal rise in serum alkaline phosphatase activity after administration of longchain fatty acids. As there is a low background of skeletal alkaline phosphatase in the serum of these patients, the intensity of the intestinal component is greatly am-

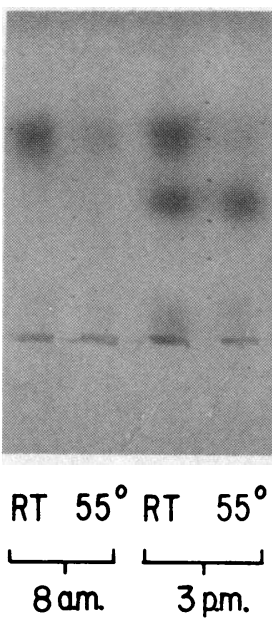

M.R.

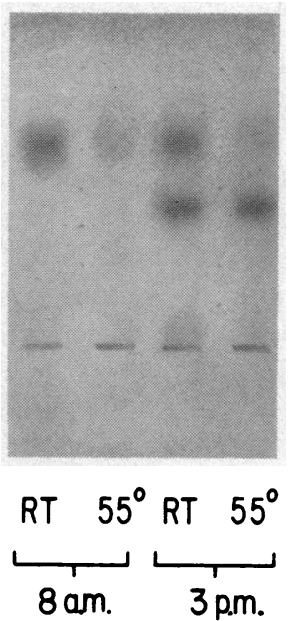

D.R.
FIGURE 3 Heat stability of serum alkaline phosphate profiles before and after administration of LCT. The 8:00 a.m. and 3:00 p.m. samples were obtained under the same conditions as in Fig. 2. Serum samples were incubated at room temperature (RT) or at $55^{\circ} \mathrm{C}$ as described in the text.

plified. It is of interest that the administration of medium-chain triglyceride did not greatly increase serum alkaline phosphatase activity. Medium-chain fatty acids are absorbed almost entirely by the portal system and thus bypass the intestinal lymph. Glickman, Alpers, Drummey, and Isselbacher (21), however, found a rapid rise of intestinal lymph alkaline phosphatase after octanoic acid administration. The failure to observe an increased serum level of alkaline phosphatase in our patients after MCT may relate to species differences or to the quantity of MCT administered. Glickman et al. (21) also demonstrated that the rise in intestinal alkaline phosphatase activity after fat administration was blocked by acetocyclohexamide, an inhibitor of protein synthesis. They concluded that protein synthesis is required for the rise in intestinal alkaline phosphatase activity after fat feeding.

It has been shown previously that patients with hypophosphatasia may not be deficient in all alkaline phosphatase activities. Danovitch et al (9), reported that patients with the condition have normal levels of intestinal alkaline phosphatase as determined on intestinal biopsy material. Newton (2) investigated serum alkaline phosphatase profiles in a number of disorders including hypophosphatasia. In one of six patients with hypophosphatasia, a slow band thought to be of intestinal origin was the principal zone of alkaline phosphatase activity. Others $(23,24)$ have demonstrated alkaline phosphatase activity in duodenal juices of patients with hypophosphatasia. 
It is also of interest that our patients had normal levels of leukocyte alkaline phosphatase. Others have reported decreased activities in hypophosphatasia $(2,25)$. This is another indication that there may be different gene loci for the defect in hypophosphatasia.

The gel profiles of serum alkaline phosphatase activity of our patients indicate that there may be other zones of intestinal alkaline phosphatase activity in addition to the major slow-moving band seen in Fig. 1. Visualization of these additional $l$-phenylalanine-sensitive fractions in the fast band and at the gel origin became more obvious after LCT feeding. Distinction of these bands was further enhanced because of the relatively low background activity of skeletal alkaline phosphatase. The source of the heat-labile alkaline phosphatase of these patients is presently not established. It may represent bone or possibly modified intestinal alkaline phosphatase (5).

One can conclude from these studies that patients with hypophosphatasia show a normal elevation in intestinal alkaline phosphatase activity after the feeding of long-chain fat. Furthermore, intestinal alkaline phosphatase may under certain nutritional conditions be the major serum alkaline phosphatase activity in hypophosphatasia.

\section{ACKNOWLEDGMENTS}

The authors are grateful for the help and cooperation of Dr. Edwin T. Wyman, Jr., of the Department of Orthopedic Surgery, Massachusetts General Hospital.

This work was supported by the John Hartford Foundation and by U. S. Public Health Service grant HD-03610.

\section{REFERENCES}

1. Rathbun, J. C. 1948. Hypophosphatasia. A new developmental anomaly. Amer. J. Dis. Child. 75: 822.

2. Fraser, D. 1957. Hypophosphatasia. Amer. J. Med. 22: 730.

3. Pimstone, B., E. Eisenberg, and S. Silverman. 1966. Hypophosphatasia: genetic and dental studies. Ann. Intern. Med. 65: 722.

4. Goyer, R. A. 1963. Ethanolamine phosphate excretion in a family with hypophosphatasia. Arch. Dis. Childhood. 38: 205 .

5. Fishman, W. H., and N. K. Ghosh. 1967. Isozymes of human alkaline phosphatase. Advan. Clin. Chem. 10: 255.

6. Posen, S. 1967. Alkaline phosphatase. Ann. Intern. Med. 67: 183.

7. Posen, S., F. C. Neale, D. J. Birkett, and J. BrudenellWoods. 1967. Intestinal alkaline phosphatase in human serum. Amer. J. Clin. Pathol. 48: 81.

8. Langman, M. J. S., E. Leuthold, E. B. Robson, J. Harris, J. E. Luffman, and H. Harris. 1966. Influence of diet on the "intestinal" component of serum alkaline phos- phatase in people of different ABO blood groups and secretor status. Nature (London). 212: 41.

9. Danovitch, S. H., P. N. Baer, and L. Laster. 1968. Intestinal alkaline phosphatase activity in familial hypophosphatasia. N. Engl. J. Med. 278: 1253.

10. Bessey, O. A., O. H. Lowry, and M. J. Brock. 1946. A method for the rapid determination of alkaline phosphatase with five cubic millimeters of serum. J. Biol. Chem. 164: 321 .

11. Fishman, W. H., and S. Green. 1967. Automated differential isoenzyme analysis. I. L-phenylalanine-sensitive isoenzymes of human serum alkaline phosphatase. Enzymologia. 33: 89.

12. Fishman, W. H., S. Green, and N. R. Inglis. 1963. Lphenylalanine: an organ-specific, stereospecific inhibitor of human intestinal alkaline phosphatase. Nature (London). 198: 685 .

13. Efron, M. L. 1966. Quantitative estimation of amino acids in physiological fluids using a Technicon amino acid analyzer. Automation in Analytical Chemistry. L. T. Skeggs, Jr., editor. Mediad, Inc., New York. 637.

14. Beck, W. S., and W. N. Valentine. 1951. Biochemical studies on leucocytes. II. Phosphatase activity in chronic lymphatic leucemia, acute leucemia and miscellaneous hematologic conditions. J. Lab. Clin. Med. 38: 245.

15. Harris, H., and E. B. Robson. 1959. A genetical study of ethanolamine phosphate excretion in hypophosphatasia. Ann. Hum. Genet. 23: 421.

16. Silverman, J. L. 1962. Apparent dominant inheritance of hypophosphatasia. Arch. Intern. Med. 110: 191.

17. Moss, D. W., and E. J. King. 1962. Properties of alkaline-phosphatase fractions separated by starch-gel electrophoresis. Biochem. J. 84: 192.

18. Beckman, L. 1968. Blood groups and serum alkaline phosphatase. Ser. Haematol. 1 : 137.

19. Blomstrand, R., and B. Werner. 1965. Alkaline phosphatase activity in human thoracic duct lymph. Acta. Chir. Scand. 129: 177.

20. Langman, M. J. S., A. Constantinopoulos, and I. A. D. Bouchier. 1968. ABO blood groups, secretor status, and intestinal mucosal concentrations of alkaline phosphatase. Nature (London). 217: 863 .

21. Glickman, R. M., D. H. Alpers, G. D. Drummey, and K. J. Isselbacher. 1970. Increased lymph alkaline phosphatase after fat feeding: effects of medium chain triglycerides and inhibition of protein synthesis. Biochim. Biophys. Acta. 201: 226.

22. Newton, M. A. 1966. A distinctive fraction of alkaline phosphatase in health and disease. J. Clin. Pathol. (London) . 19: 491 .

23. Scaglione, P. R., and J. F. Lucey. 1956. Further observations on hypophosphatasia. J. Dis. Child. 92: 493.

24. Davidson, R. G., R. Davidson, L. L. Mosovich, and S. J. Yaffe. 1969. Hypophosphatasia. A developmental anomaly of alkaline phosphatase. The Hague Conference on Birth Defects. September, 1969. Excerpta Med. Int. Congr. Ser. 191: 35.

25. Kretchmer, N., M. Stone, and C. Bauer. 1958. Hereditary enzymatic effects as illustrated by hypophosphatasia. Ann. N. Y. Acad. Sci. 75: 279. 